\title{
Tensión superficial, viscosidad y densidad de algunas escorias $\mathrm{CaO}-\mathrm{Al}_{2} \mathrm{O}_{3}^{(\cdot)}$
}

\author{
F.A. Oliveira ${ }^{(*)}$, A. Miller ${ }^{(* *)}$ y J. Madías ${ }^{(* * *)}$
}

\begin{abstract}
Resumen Los conceptos metalúrgicos con respecto a la estructura y propiedades de los aluminatos de calcio, están predominantemente basados en analogías con el sistema ternario $\mathrm{CaO}-\mathrm{Al}_{2} \mathrm{O}_{3}-\mathrm{SiO}_{2}$. En este trabajo se presentan y discuten los resultados de medidas de tensión superficial, densidad y viscosidad de algunas escorias de aluminato de calcio, en la franja de temperatura entre 1.500 y $1.650{ }^{\circ} \mathrm{C}$, a la luz de investigaciones recientes sobre caracterización de la estructura vítrea de aluminatos y alúmino-silicatos. La faja de composición investigada fue de 45 a $52 \%$ en peso de alúmina. Los resultados indican una reducción en la densidad con la disminución de la razón molar $\mathrm{CaO}: \mathrm{Al}_{2} \mathrm{O}_{3}$. La tensión superficial disminuye con el aumento de la relación molar $\mathrm{CaO}: \mathrm{Al}_{2} \mathrm{O}_{3}$ o con el aumento de la temperatura, en tanto que la viscosidad aumenta con la elevación del contenido de alúmina y disminuye con el aumento de la temperatura. El comportamiento observado en los valores de tensión superficial y viscosidad con la variación de la composición, puede estar asociado a la presencia de algunos iones de aluminio en coordinación octaédrica que, a su vez, pueden presentar una proporción de aluminatos conteniendo iones de aluminio en reducido estado de valencia, tal como $\mathrm{Al}^{2+}$.
\end{abstract}

Palabras clave: Escoria. Aluminato de calcio. Estructura. Propiedades físico-químicas.

\section{Surface tension, densities and viscosities of some $\mathrm{CaO}-\mathrm{Al}_{2} \mathrm{O}_{3}$ slags}

\begin{abstract}
The metallurgical concepts with regard to the structures and properties of calcium aluminate melts have been based upon analogies within ternary $\mathrm{CaO}-\mathrm{Al}_{2} \mathrm{O}_{3}-\mathrm{SiO}_{2}$ systems. In this work the results of surface tension, density and viscosity of some calcium aluminate slags, in the temperature range of 1500 to $1600{ }^{\circ} \mathrm{C}$ are presented and the results are discussed based upon recent structural characterisation results of crystalline aluminates. The compositional range investigated was from 45 to 53 weight- $\%$ alumina. The results indicate a reduction in density as the molar ratio $\mathrm{CaO}-\mathrm{Al}_{2} \mathrm{O}_{3}$ decreases. Surface tension falls on increasing either the molar ratio $\mathrm{CaO}-\mathrm{Al}_{2} \mathrm{O}_{3}$ or temperature. Conversely, viscosity increases with increasing molar ratio $\mathrm{CaO}-\mathrm{Al}_{2} \mathrm{O}_{3}$ and decreasing temperature. The compositional dependence of both surface tension and viscosity data may be associated with the presence of some aluminium ions in octahedral co-ordination, and a concept of surface behaviour is proposed which involves surface activity of aluminate anions containing aluminium ions in a reduced valence state, such as $\mathrm{Al}^{2+}$.
\end{abstract}

Keywords: Slags. Calcium aluminates. Structure. Physicochemical properties.

\section{INTRODUCCIÓN}

Los aluminatos son importantes como materiales refractarios y escorias metalúrgicas. Escorias alumi-

(•) Trabajo recibido el día 5 de octubre de 1998 y aceptado en su forma final el día 8 de abril de 1999.

(*) Escuela Politécnica da Universidade Federal da Bahia, EPUFBA; Rua Ubaldo Osório, 8 - Federação, CEP 40210-630 Salvador, BA, Brasil.

(**) Manchester Materials Science Centre; Grosvenor Street, Manchester, M1 7HS (Inglaterra).

(***) Instituto Argentino de Siderurgia, Av. Central y Calle 19 Oeste, 2900 San Nicolás, Provincia de Buenos Aires (República Argentina). nosas se emplean comúnmente tanto en operaciones pirometalúrgicas, como en procesos de desulfuración y afino del acero líquido y como base de escorias industriales, empleadas en la reducción y afino de metales no férreos. También en la metalurgia de cuchara y en la colada continua de los aceros calmados al aluminio y tratados con calcio, dada la presencia de inclusiones de aluminato de calcio que juegan un papel central en la colabilidad y en algunas propiedades del acero, el conocimiento de sus propiedades físicas es de especial interés.

El estado actual del conocimiento de la estructura y la termodinámica de las fases de aluminatos líquidos es todavía incompleto, aunque suficiente 
para diversas aplicaciones de carácter práctico. Estructuralmente, se asume que, semejantes a silicatos líquidos, los aluminatos poseen ajustes estructurales de aniones en diferentes grados de polimerización. A pesar de la constatación de un considerable progreso sobre la constitución líquida de aluminatos, dificultades de interpretación de sus propiedades físico-químicas han ocasionado discrepancias en la literatura metalúrgica. La determinación de la dependencia estructural de sus propiedades físicoquímicas es exacerbada por dificultades experimentales, asociadas al elevado punto de fusión y naturaleza corrosiva de su fase líquida. A pesar de las incertidumbres experimentales, existe un consenso general sobre las tendencias de los resultados.

Mediante resultados de espectroscopía y difracción de rayos $\mathrm{X}$ de materiales vítreos de aluminatos y silicatos de aluminio se pueden obtener valiosas informaciones sobre la estructura de los aluminatos. Asumiendo que bajas entropías de fusión indican ajustes estructurales mínimos, se considera que a través de una práctica eficiente de congelamiento, la estructuración vítrea se aproxima a la que presenta en fusión. Estas prácticas, aliadas a las técnicas actuales de caracterización estructural, permiten establecer analogías consistentes entre la estructura cristalina y vítrea de un sistema específico.

Todavía, el actual grado de conocimiento de la estructura de escorias en el estado líquido, imposibilita el desarrollo de modelos termodinámicos completos de estructuras, particularmente con relación a escorias alúmino-siliciosas. Estos modelos, normalmente asumen los aniones siliciosos como agrupamiento estructural dominante. Sin embargo, el mecanismo de sus interacciones con otros aniones formadores de red es desconocido, en especial con la alúmina. Esto puede ser consecuencia de dos hechos: 1) la naturaleza anfótera de la alúmina, que disminuye la actividad de la sílice en soluciones ricas en sílice y eleva ligeramente la actividad de la sílice en soluciones básicas; y 2) el ion $\mathrm{Al}^{3+}$ es capaz de substituir directamente el $\mathrm{Si}^{4+}$ en estructuras tetraédricas de silicatos, pero su comportamiento en soluciones ricas en alúmina es poco comprendido.

Algunas interpretaciones concretas de las propiedades y estructura de escorias alúmino-siliciosas, pueden provenir de informaciones con respecto a los efectos del silicio en la estructuración vítrea y propiedades físico-químicas de aluminatos.

El presente trabajo muestra los resultados experimentales de medidas de densidad, tensión superficial y viscosidad en escorias aluminosas, conteniendo 46 a $55 \% \mathrm{CaO}$ en peso. Se discute la dependencia de las propiedades físico-químicas respecto de las transformaciones estructurales, a la luz de recientes investigaciones de caracterización estructural de los aluminatos de calcio.

\section{METODOLOGÍA EXPERIMENTAL}

\subsection{Densidad y tensión superficial}

Las muestras de escorias para determinación de tensión superficial y densidades fueron preparadas a partir de polvos de reactivos puros para análisis, óxido de aluminio Analar y carbonato de calcio. Los polvos fueron pesados, mezclados en un mezclador rotativo durante $2 \mathrm{~h}$ y posteriormente briqueteados y almacenados en estufa. Las briquetas fueron luego cargadas en crisoles de molibdeno de $100 \mathrm{~mm}$ de altura por $60 \mathrm{~mm}$ de diámetro exterior. La carga se fundió bajo atmósfera de argón, en un horno de resistencia de grafito, el mismo que se empleó para la realización de medidas de tensión superficial.

Para determinar la tensión superficial y las densidades de las escorias líquidas se empleó el método de Máxima Presión de Burbuja (1). El aparato experimental está presentado esquemáticamente en la figura 1.

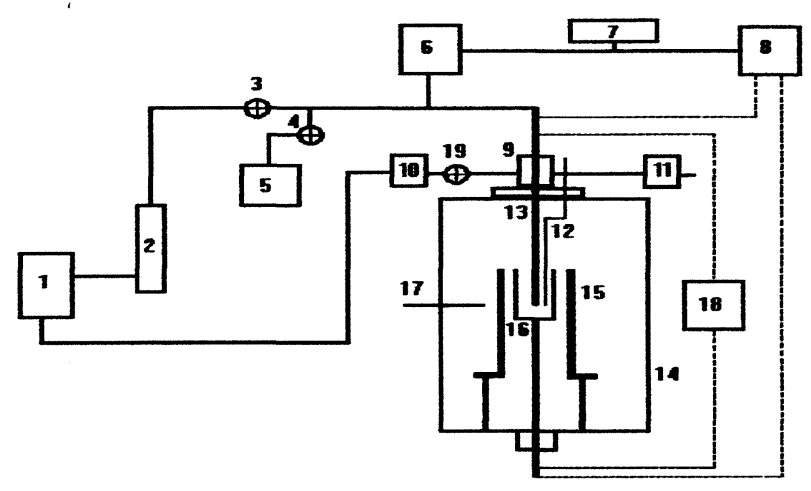

FIG. 1.- Equipamiento para determinación de la tensión superficial a alta temperatura:

1- Cilindro de Argón. 2- Rotámetro. 3-Válvula aguja. 4- Válvula by-pass. 5- Manómetro. 6- Medidor de presión. 7- Milivoltímetro indicador digital. 8- Fuente eléctrica (DC). 9- Sello "Wilson". 10- Rotámetro. 11- Salida de gas. 12-Termopar PtRh. 13- Capilar de molibdeno. 14- Horno. 15- Elemento de resistencia (grafito). 16- Crisol de molibdeno. 17- Termopar W-Re. 18- Voltímetro digital. 19- Controlador de caudal (Ar).

FIG. 1.- Maximum bubble experimental apparatus scheme:

1- Argon supply. 2- Flow meter. 3- Needle valve. 4.- Manometer bypass valve. 5.- Manometer. 6.- Pressure transducer. 7.- Digital voltmeters. 8.- DC power. 9.- Wilson seal. 10.- Flow meter (gas purge to furnace). 11.- Gas bubble (furnace outlet). 12.- Pt-Rh thermocouple. 13.- Molybdenum cappilary. 14.- Furnace. 15.- Graphite heating element. 16.- Molybdenum crucible. 17.- W-Re thermocouple. 18.- Digital voltmeters. 19.- Gas purge valve 
En este método, se fuerza la formación lenta de burbujas de argón en el lecho líquido de las escorias, a través de capilares de molibdeno, $(500 \mathrm{~mm}$ de longitud por $3 \mathrm{~mm}$ de diámetro interior).

La máxima presión de gas requerida para liberar la burbuja cambia linealmente con la profundidad de inmersión del capilar. Esta presión se iguala a la suma de la presión hidrostática de la columna de escoria líquida y de la presión requerida para crear una nueva superficie contra las fuerzas de tensión superficial. Experimentalmente, la máxima presión de la burbuja se mide en términos de una correspondiente columna de líquido (ftalato de n-dibutilo), en un manómetro conectado al tubo capilar. Las tensiones superficiales se calculan con el uso de la fórmula de Schrödinger (2),

$$
\begin{gathered}
\sigma=\left(r P_{\sigma} / 2\right)\left[\left(1-(2 / 3)\left(r \rho g / P_{\sigma}\right)-\right.\right. \\
\left.-(1 / 6)\left(r \rho g / P_{\sigma}\right)^{2}\right]
\end{gathered}
$$

donde $\sigma$ es la tensión superficial $\left(\mathrm{mN} \cdot \mathrm{m}^{-1}\right), r$ es el radio interno del capilar $(\mathrm{m}), P_{\sigma}$ es la presión necesaria para crear una nueva superficie contra la tensión superficial, o sea $P_{\max }-\rho g h\left(\mathrm{~N} \cdot \mathrm{m}^{-2}\right), \rho$ es la densidad de la escoria $\left(\mathrm{g} \cdot \mathrm{m}^{-3}\right)$ y $\mathrm{g}$ la aceleración de la gravedad $\left(\mathrm{m} \cdot \mathrm{s}^{-2}\right)$.

Medidas simultáneas de densidad fueron realizadas y obtenidas a través de la relación entre la variación de la presión máxima, $P$, con la profundidad de inmersión, $h$, dada por;

$$
\rho=(1 / g)(\delta P / \delta h)_{\mathrm{T}}
$$

donde $g$ es la constante gravitacional $\left(\mathrm{kg} \cdot \mathrm{m}^{-2}\right)$
Mayores detalles del método experimental empleado y análisis de los errores experimentales involucrados han sido publicados anteriormente (3a). El aparato experimental se presenta esquemáticamente en la figura 1.

Durante la fusión se observó una prolongada efervescencia, siendo necesaria la aplicación de períodos isotérmicos de $4 \mathrm{~h}$ a $1.600^{\circ} \mathrm{C}$, antes de la realización de las mediciones, con el objetivo de lograr una completa quietud de la superficie líquida de las escorias. Dificultades en la localización de la superficie líquida de las escorias ocasionaron una variación experimental en los resultados obtenidos del orden de un $5 \%$, con un nivel de confianza del $95 \%$. Al final de cada experiencia, se extraían muestras de escorias y se analizaban por medio de fluorescencia de rayos X (Tabla I).

\subsection{Viscosidad}

Las muestras para mediciones de viscosidad se prepararon por mezcla de reactivos de alúmina y óxido de calcio de grado analítico. Las mezclas homogeneizadas se cargaron en crisoles de molibdeno, en cantidades de $10 \mathrm{~g}$, y posteriormente se fundieron en un horno de inducción, en atmósfera inerte de argón.

Este procedimiento se repitió hasta que los crisoles poseyeran $100 \mathrm{~g}$ de material completamente fundido. Durante la etapa de fusión, se registró la aparición de espumas (slag foaming), particularmente en la fusión de mezclas con un contenido del $53 \%$ en peso de alúmina. Esto se debió a una

\begin{tabular}{|c|c|c|c|c|}
\hline \multicolumn{5}{|c|}{ Muestras empleadas para la determinación de viscosidad } \\
\hline \multirow{2}{*}{ Escorias } & \multicolumn{2}{|c|}{ Composiciones calculadas } & \multicolumn{2}{|c|}{ Resultados análisis FRX } \\
\hline & $\mathrm{CaO}, \%$ & ${ }_{2} \mathrm{O}_{3}, \%$ & $\mathrm{CaO}, \%$ & $\mathrm{Al}_{2} \mathrm{O}_{3}, \%$ \\
\hline 1 & 55 & 45 & $53,0(54,1)$ & $45,0(45,9)$ \\
\hline 2 & 52 & 48 & $49,0(50,9)$ & $47,2(49,0)$ \\
\hline 3 & 50 & 50 & $45,9(48,8)$ & $50,6(51,1)$ \\
\hline 4 & 48 & 52 & $45,9(47,0)$ & $51,6(52,9)$ \\
\hline 5 & 46 & 54 & 44,8 & 54,8 \\
\hline \multicolumn{5}{|c|}{ Muestras empleadas para la determinación de la tensión superficial } \\
\hline 6 & 55 & 45 & 49,5 & 49,9 \\
\hline 7 & 52 & 48 & $50,7(51,7)$ & $47,4(48,3)$ \\
\hline 8 & 50 & 50 & 46,4 & 52,9 \\
\hline 9 & 48 & 52 & 47,3 & 52,1 \\
\hline 10 & 47 & 53 & $45,6(46,3)$ & $52,7(53,6)$ \\
\hline 11 & 46 & 54 & $40,7(45,4)$ & $48,8(54,5)$ \\
\hline
\end{tabular}

TABLA I.- Análisis de las muestras de escorias. Resultados normalizados

TABLE I.- Analysis of slag samples. Normalized results 
prolongada efervescencia observada durante la fusión. Solamente luego de observar quietud de la superficie de la escoria, por un período de unos pocos minutos, el crisol y su contenido eran enfriados a la temperatura ambiente, en atmósfera de argón.

Para las mediciones de viscosidad a alta temperatura se utilizó un viscosímetro tipo Brookfield LVTD, con atmósfera controlada de argón. Tanto el crisol como el cilindro coaxial empleados en las medidas eran de molibdeno. El cilindro fue mantenido a una altura de $10 \mathrm{~mm}$ de la base del crisol, siendo completamente cubierto con escoria líquida fundida. Se realizaron experiencias preliminares con aceites patrones y escorias para evaluar el funcionamiento del viscosímetro, (4).

Las muestras fueron sometidas a tratamiento isotérmico a $1.600^{\circ} \mathrm{C}$, durante $4 \mathrm{~h}$, antes de la realización de las mediciones. Las incertidumbres experimentales se estimaron en $< \pm 10 \%$. Las muestras enfriadas a la temperatura ambiente, con una velocidad de $2{ }^{\circ} \mathrm{C} \cdot \mathrm{min}^{-1}$, fueron luego analizadas por fluorescencia de rayos X (Tabla I).

\section{RESULTADOS}

Los análisis de las escorias utilizadas en los ensayos de viscosidad, tensión superficial y densidad se presentan en la tabla I, donde se comparan con los resultados de los análisis realizados.

En general, se confirma que las composiciones obtenidas coinciden con las calculadas. Se observa que la relación entre las composiciones teóricas y las analizadas, fue mejor para los muestreos realizados después de los ensayos de viscosidad.

Los resultados de viscosidades, obtenidos para los rangos de temperaturas entre 1.330 y $1.600^{\circ} \mathrm{C}$, están presentados en la tabla II. La energía de activación del fluido viscoso se obtuvo asumiendo una relación lineal (Arrhenius-Gusman) entre $\log \eta$ frente a $1 / T$.
TABLA II.- Viscosidad y energía de activación del fluido viscoso a $1.550^{\circ} \mathrm{C}$

TABLE II. - Viscosity and activation energy of viscous fluid at $1.550^{\circ} \mathrm{C}$

\begin{tabular}{|c|c|c|}
\hline $\begin{array}{c}\text { Composición } \\
\left(\mathrm{Al}_{2} \mathrm{O}_{3}, \%\right)\end{array}$ & Viscosidad & $\begin{array}{c}\text { Energía de } \\
\text { activación } \\
\left.\text { (kJ.moles }^{-1}\right)\end{array}$ \\
\hline 45 & 0,24 & 87 \\
48 & 0,28 & 98 \\
50 & 0,34 & 94 \\
52 & 0,59 & 201 \\
53 & 1,90 & 134 \\
4 & 0,40 & 98 \\
\hline
\end{tabular}

Los resultados revelan que la viscosidad disminuye con el contenido de $\mathrm{CaO}$ y la temperatura, de manera similar a las tendencias observadas para líquidos binarios de $\mathrm{CaO}-\mathrm{SiO}_{2}$ (5). La dependencia de la viscosidad con la composición para este sistema, sugiere un aumento de la viscosidad con el aumento de la alúmina. Por analogía con el comportamiento de la sílice en silicatos binarios en líquidos binarios básicos, se pone en evidencia la capacidad de la alúmina en actuar como anión formador de red.

Los resultados de densidades y tensiones superficiales para las escorias líquidas de aluminato de calcio, están presentados en las tablas III y IV.

Los valores de las densidades, determinados experimentalmente, poseen coherencia con los resultados publicados por la literatura (6 y 7), como también tienen una tendencia similar a la predicha por el modelo de Mills (8). Los resultados experimentales indican una reducción de la densidad con la concentración de alúmina. Sin embargo, no

TABLA III.- Resultados de densidades de aluminatos de calcio

TABLE III.-Results of densities of calcium aluminates

\begin{tabular}{|c|c|c|c|c|c|c|}
\hline \multirow[b]{2}{*}{$\mathrm{Al}_{2} \mathrm{O}_{3}, \%$} & \multicolumn{5}{|c|}{ Densidad $(\mathrm{g} / \mathrm{ml})$} & \multirow{2}{*}{$\begin{array}{l}\text { Modelo } \\
1.550^{\circ} \mathrm{C}\end{array}$} \\
\hline & $1.570^{\circ} \mathrm{C}$ & $1.580^{\circ} \mathrm{C}$ & $1.585^{\circ} \mathrm{C}$ & $1.595^{\circ} \mathrm{C}$ & $1.610^{\circ} \mathrm{C}$ & \\
\hline 45 & 2,82 & 2,82 & - & 2,84 & - & 2,79 \\
\hline 48 & 2,78 & 2,84 & 一 & 2,82 & - & 2,78 \\
\hline 50 & 2,82 & 2,81 & - & 2,81 & - & 2,78 \\
\hline 52 & 2,77 & 2,77 & - & 2,76 & - & 2,78 \\
\hline 53 & 2,73 & 2,72 & - & 2,77 & - & 2,78 \\
\hline 54 & - & - & 2,77 & 2,76 & 2,78 & 2,77 \\
\hline
\end{tabular}


TABLA IV.- Tensión superficial de aluminatos de calcio

TABLE IV.- Surface tension of calcium aluminates

\begin{tabular}{|c|c|c|c|c|c|c|}
\hline \multirow[b]{2}{*}{$\mathrm{Al}_{2} \mathrm{O}_{3}, \%$} & \multicolumn{5}{|c|}{ Tensión superficial $\left(\mathrm{mN} \cdot \mathrm{m}^{-1}\right)$} & \multirow{2}{*}{$\begin{array}{l}\text { Modelo } \\
1.550{ }^{\circ} \mathrm{C}\end{array}$} \\
\hline & $1.570{ }^{\circ} \mathrm{C}$ & $1.580{ }^{\circ} \mathrm{C}$ & $1.585^{\circ} \mathrm{C}$ & $1.595^{\circ} \mathrm{C}$ & $1.610^{\circ} \mathrm{C}$ & \\
\hline 45 & 534,8 & 532,5 & - & 524,1 & - & 634,3 \\
\hline 48 & 555,5 & 540,2 & - & 535,5 & - & 635,1 \\
\hline 50 & 561,1 & 549,2 & - & 539,4 & - & 635,6 \\
\hline 52 & 564,1 & 551,4 & - & 536,9 & - & 636,2 \\
\hline 53 & 608,4 & 564,5 & - & 540,5 & - & 636,5 \\
\hline 54 & - & - & 555,9 & 550,5 & 548,4 & 636,8 \\
\hline
\end{tabular}

muestran coeficientes de expansión consistentes para el rango de temperatura investigado.

Los resultados de la tabla III pueden ser interpretados en términos de la contribución molar parcial de ambos óxidos, al volumen parcial molar del sistema. El volumen molar parcial de la alúmina, estimado en $28,96 \mathrm{~cm}^{3} \cdot \mathrm{mol}^{-1}$, se presenta superior al del óxido de calcio, $21 \mathrm{~cm}^{3} \cdot \mathrm{mol}^{-1}$.

Con base en estos valores, la densidad disminuye proporcionalmente al descenso de la razón molar $\mathrm{CaO}: \mathrm{Al}_{2} \mathrm{O}_{3}$, sugiriendo que el aumento en la fracción molar de alúmina ocasiona el descenso de la densidad de la escoria líquida.

Sin embargo, y aún sin considerar las complejas interacciones entre estructuras, volumen parcial molar y composición, esta hipótesis deviene en una previsión inadecuada de la dependencia de la densidad de sistemas binarios con respecto a la composición.

Se hace difícil una discusión más amplia de los resultados de la densidad, en términos del volumen molar parcial de los óxidos involucrados, debido al limitado rango de composiciones investigado.

Los resultados experimentales presentados en la tabla IV indican un aumento de la tensión superficial con el aumento en el contenido de alúmina. Esta dependencia de la tensión superficial respecto a la composición de las escorias investigadas, a pesar de estar confirmada por resultados publicados anteriormente (9), no fue explicada hasta entonces.

La tensión superficial puede considerarse como una medida de la concentración de uniones no saturadas, de moléculas o de átomos, en la superficie de líquidos o sólidos. En silicatos binarios líquidos la tensión superficial aumenta con la basicidad, reflejando la despolimerización del interior de la fase líquida. Se sugiere que aniones formadores de red $\left(\mathrm{SiO}^{2-}\right)$, tienden a disminuir la tensión superficial debido a que se concentran preferencialmente próximos a la superficie. En contrapartida, los aniones de silicato pueden polimerizar la superficie y con- tribuir al aumento de la viscosidad superficial a causa de esa misma polimerización.

Las alteraciones de la tensión superficial con respecto a la composición, pueden analizarse en términos de la ecuación de adsorción de Gibbs, que en su forma reducida

$$
\text { - } d \sigma=\Gamma_{\mathrm{S}} \quad \mathrm{x} d \mu_{\text {soluto }}
$$

predice una concentración superficial positiva en exceso de soluto, $\Gamma_{\mathrm{S}}$, si su adición produce una disminución en la tensión superficial, $\sigma$. La aplicación práctica de la ecuación [3] confirma algunas sugerencias acerca de una reducción en la tensión superficial de silicatos, boratos y fosfatos binarios con el aumento de la actividad de sus respectivos grupos aniónicos (10). Las adiciones de un modificador de red, tal como el $\mathrm{CaO}$, en la estructura polimérica tetraédrica de aluminatos, deben promover la despolimerización y, en consecuencia, una disminución en la tensión superficial. Esto podría explicar los resultados presentados en la tabla IV. Sin embargo, esta tendencia no está confirmada.

Con base en estos fundamentos de química de superficie, los resultados experimentales presentados en la tabla IV permiten suponer que la estructura de las escorias aluminosas líquidas se torna totalmente despolimerizada en el rango de composición investigado. Se puede concluir que la alúmina presenta un comportamiento anómalo, para lo que debería esperarse en relación con el comportamiento de otros aniones formadores de red en sistemas binarios. Este comportamiento justifica la necesidad de investigaciones más completas sobre la estructura y la termodinámica del sistema $\mathrm{CaO}$ $\mathrm{Al}_{2} \mathrm{O}_{3}$.

A pesar de que se constatan algunas divergencias sobre el comportamiento estructural de la alúmina en resultados de investigaciones de caracterización estructural de materiales vítreos, pueden 
obtenerse muchas inferencias del diagnóstico de la sensibilidad estructural de las propiedades físicoquímicas de las escorias alúmino-siliciosas (3b).

\section{DISCUSIÓN DE LOS RESULTADOS}

Los resultados obtenidos para la viscosidad confirman un aumento de la misma a medida que aumenta la concentración de alúmina, en el rango de composiciones investigado, y pueden estar reflejando la facultad de la alúmina para actuar como un anión formador de red. Asumiendo que estos resultados reflejan recíprocamente la viscosidad superficial, se infiere un relativo aumento del grado de polimerización en la superficie y consecuentemente, en la concentración de especies aniónicas de aluminatos.

Las mediciones de viscosidad se efectuaron rigurosamente después de una acentuada supresión de espumas o efervescencia. Se descarta así la posibilidad de que los efectos causados por la efervescencia, en las muestras que contienen 52 y $53 \% \mathrm{M}$ de alúmina, se reflejen en un aparente aumento en la energía de activación del fluido viscoso. La efervescencia de las escorias líquidas se registró particularmente para muestras con composición próxima a fases que comprendían el compuesto $12 \mathrm{CaO} 7 \mathrm{Al}_{2} \mathrm{O}_{3}$. Esta composición sugiere la existencia de concentraciones de super-radicales de oxígeno, $\mathrm{O}_{2}^{-}$, y efervescencia asociada, debido a su liberación durante la fusión (11).

El valor de la tensión superficial de las escorias investigadas confirma una tendencia anómala del aumento en la tensión superficial con la concentración de alúmina. A pesar de que esta tendencia se asemeja a resultados previamente publicados (9), no se explica por analogía con otros sistemas binarios. Desde que el comportamiento de la alúmina en el interior de escorias líquidas es comparable al de otros aniones formadores de red, se percibe una discrepancia entre las propiedades de la alúmina en el interior de aluminatos líquidos, en relación a sus propiedades superficiales.

La capacidad de los iones $\mathrm{Ca}^{2+}$ para comportarse como especies activadoras de superficie, aunque adversa al comportamiento experimentalmente comprobado de la actividad superficial de grupos aniónicos, puede evaluarse mediante la medición de la concentración en exceso de $\mathrm{CaO}$ en la superficie, $\left(\Gamma_{\mathrm{Al}_{2} \mathrm{O}_{3} \mathrm{CaO}}\right)$, de acuerdo con la ecuación [3]. En caso dé saturación de la superficie, se asume que el área cubierta es similar a la ocupada por un átomo, en un plano dado de su célula unitaria. La superficie en exceso calculada a $1.595{ }^{\circ} \mathrm{C}$, fue de $1,38 \cdot 10^{-10} \mathrm{M} \cdot \mathrm{cm}^{-2}$. Este valor no equivale al área de un átomo libre de calcio o a algún plano cristalográfico del $\mathrm{CaO}$.
Con estos resultados, se cuestiona la aplicabilidad de la isoterma de adsorción de Gibbs a líquidos iónicos. Su formalismo asume como base que la interfaz es un plano matemático entre fases y sin volumen (12). Una interfaz debe poseer un espesor finito y un volumen, donde las propiedades de un sistema se alteran gradualmente de una fase a otra. Este hecho, reconocido como un modelo alternativo (13), torna la superficie en una región de transición. Según esto, se infiere que la superficie de líquidos iónicos puede estar constituida por una asociación de aniones con cationes, en la forma de una doble camada eléctrica.

Por lo tanto, se vuelve discutible interpretar la tensión superficial en términos de una única especie en exceso, particularmente si esta especie es una fase cristalina y eléctricamente neutra, como el $\mathrm{CaO}$.

A partir de informaciones sobre la estructura vítrea de los aluminatos de calcio, se pueden generar interpretaciones alternativas del aumento en la tensión superficial con la concentración de alúmina. En 'la relación molar $\mathrm{CaO}: \mathrm{Al}_{2} \mathrm{O}_{3}=1$, la estructura de estos aluminatos está constituida básicamente de una red tetraédrica de alúmina, totalmente polimerizada. Para relaciones molares por encima o por debajo de la equimolar, se produce un aumento desordenado de la coordinación del $\mathrm{Al}^{3+}$, con aumento de oxígeno libre en grupos aniónicos. Esto sugiere una tensión superficial mínima para composiciones en la relación equimolar.

Así, en las relaciones molares $\mathrm{CaO}: \mathrm{Al}_{2} \mathrm{O}_{3}<1$, las adiciones de alúmina al monoaluminato de calcio reducen la media de oxígenos disponibles para cada aluminio. Con progresivos aumentos en la concentración de alúmina, la red polimérica tetraédrica de alúmina se reacomoda, adoptando la formación de una red de grandes poliedros coordinados octaédricamente.

Para relaciones $\mathrm{CaO}: \mathrm{Al}_{2} \mathrm{O}_{3}>1$, las adiciones de $\mathrm{CaO}$ al monoaluminato de calcio introducen un exceso de oxígenos a la red polimérica tetraédrica de la alúmina, quitándole estabilidad. Con el aumento de la basicidad y, consecuentemente, la disminución en la concentración de aniones formadores de red, se impulsa un aumento en el número de oxígenos libres asociados a los grupos aniónicos de aluminatos.

En estas condiciones, se sugiere la adopción de números de coordinación más altos, inclusive octaédricos, por parte de algunos de los iones de aluminio (14). Esta tendencia es pertinente al rango de composición de 31 - $40 \% \mathrm{M}$ de alúmina, el cual incorpora todo el rango de composición investigado.

Aparentemente, los iones $\mathrm{Al}^{3+}$ coordinados octaédricamente, podrían comportarse como modificadores de red y así ocasionar aumentos en la tensión 
superficial, por medio de una despolimerización de la superficie de la alúmina. Por otro lado, iones $\mathrm{Al}^{3+}$ coordinados tetraédricamente, se comportan como formadores de red y tienden a disminuir la tensión superficial.

Para el rango de composición investigado, los resultados de la espectroscopía sugieren que los iones $\mathrm{Al}^{3+}$ poseen variados estados de coordinación, 4, 5 o 6, (14). La tendencia observada en la variación de la tensión superficial podría ocasionarse por la presencia en la superficie, de aluminio en niveles de coordinación mayores que 4 . Con todo, esto requiere que la alúmina se comporte como modificador de red para el rango completo de composición investigado $\left(45-55 \% \mathrm{Al}_{2} \mathrm{O}_{3}\right)$, lo que es incompatible con los valores de viscosidad obtenidos.

Desde que la presencia de grupos aniónicos con uniones libres de oxígenos contribuye a aumentar la tensión superficial, un análisis de la sensibilidad a la presión parcial de oxígeno de las escorias aluminosas puede ser relevante para los resultados obtenidos. En estos ensayos, la efervescencia promovió dificultades experimentales, dificultando la determinación de la superficie líquida de las escorias. Variados grados de efervescencia fueron observados para todas las composiciones investigadas. Se adoptaron largos tiempos de equilibrio, entre 8 y 72 $\mathrm{h}$, antes de que la superficie fuera detectada con reproducibilidad.

Los valores de densidad y tensión superficial publicados equivalen al de equilibrio. Sin embargo, la extensión de su dependencia sobre la presión parcial de oxígeno no es ampliamente comprendida. Se observó que la efervescencia no se restringió a composiciones exactamente equivalentes a las del compuesto $12 \mathrm{CaO} \cdot 7 \mathrm{Al}_{2} \mathrm{O}_{3}$, como ha sido ampliamente divulgado por diversos autores. Esta afirmación puede reforzar la incertidumbre respecto al diagrama de fases $\mathrm{CaO}-\mathrm{Al}_{2} \mathrm{O}_{3}$ (15) y con el equilibrio de fases en relación con el potencial de oxígeno (16).

El desprendimiento del oxígeno por las escorias permite prever la necesidad de un reajuste estructural de equilibrio. Los disturbios estructurales involucrados probablemente afectarían a los iones de aluminio y de modo más pronunciado a los posicionados en la interfaz líquido-gas. El mantenimiento de la neutralidad eléctrica debe dar como resultado la concentración de oxígenos libres y/o la adopción de estados de valencia reducidos, para una porción de los cationes de aluminio.

Durante la investigación de la cinética de transferencia de azufre entre gases y escorias aluminosas líquidas, se ha observado evidencia adicional de los efectos del potencial de oxígeno sobre los aluminatos líquidos (17). Se deduce que con la reducción del potencial de oxígeno, una pequeña pero crecien- te proporción de azufre fue capaz de reaccionar con dos oxígenos y fue removido de la cadena aniónica de la siguiente forma;

$$
\begin{gathered}
\left(\mathrm{Al}_{2} \mathrm{SO}_{2}\right)^{2-}+\left(\mathrm{O}^{2-}\right)+\mathrm{O}_{2}(\mathrm{~g})= \\
=\left(\mathrm{Al}_{2} \mathrm{O}_{5}\right)^{4-}+1 / 2 \mathrm{~S}_{2}(\mathrm{~g})
\end{gathered}
$$

Este azufre, en estado aparentemente anómalo, participa de las reacciones de sulfuración/desulfuración a través de los siguientes mecanismos;

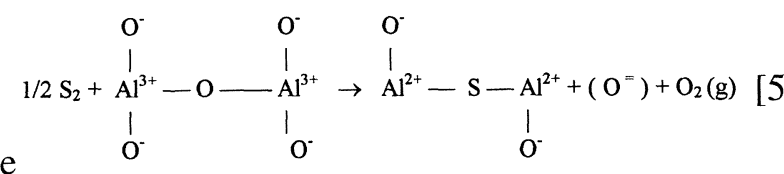

$$
\begin{aligned}
& \begin{array}{ccc}
\mathrm{O}^{-} & \mathrm{O}^{-} & \mathrm{O}^{-} \\
\mathrm{Al}^{2^{2+}}-\mathrm{S}-\mathrm{Al}^{2+}+\left(\mathrm{O}^{=}\right)+\mathrm{O}_{2}(\mathrm{~g}) & \stackrel{\mathrm{Al}^{3+}}{l^{2}}-\mathrm{O}-\mathrm{Al}^{3+}+1 / 2 \mathrm{~S}_{2}(\mathrm{~g}) \\
\mathrm{O}^{-} & \mathrm{O}^{-} & \mathrm{O}^{-}
\end{array}
\end{aligned}
$$

Se concluyó que el aluminio bivalente puede existir en asociación con el azufre en la red aniónica de las escorias aluminosas. A pesar de ser poco común, el concepto de iones de aluminio adoptando valencia $2+$ en escorias, permite solucionar discrepancias encontradas en la literatura, principalmente con respecto a la solubilidad del azufre en los aluminatos de calcio.

A este respecto, Richardson (18), informó que la solubilidad del azufre en aluminatos de calcio aumenta con la concentración de $\mathrm{CaO}$, en tanto que Cameron (19) sugiere que ésta disminuye. Las principales diferencias entre estas investigaciones se refieren al potencial de oxígeno empleado $y$, aparentemente, sus discrepancias pueden resolverse si la concentración de azufre en el estado anómalo muestra la misma proporcionalidad al $\mathrm{PO}_{2}$ y a la actividad de la alúmina.

Esto es importante para la práctica metalúrgica de procesos de desulfuración, de control de la distribución del azufre entre el metal y la escoria, así como para las investigaciones en cuanto al efecto del azufre sobre la actividad del silicio y el aluminio en escorias alúmino-siliciosas.

La capacidad del aluminio para adoptar un estado de valencia reducido en fases condensadas fue postulada para explicar los resultados de difracción sobre la composición y la estructura del plano (0001) de la alúmina- $\alpha$ (20). Se deduce que durante el tratamiento térmico a temperaturas superiores a $1.250{ }^{\circ} \mathrm{C}$, la estructura superficial de la alúmina- $\alpha$ se reacomoda y se transforma en una nueva estructura, deficiente en oxígeno.

Esta transformación, acompañada por el simultáneo desprendimiento de oxígeno, es reversible y dependiente de la presión parcial de oxígeno por encima de la superficie. Se deduce, además, que los 
cationes de aluminio en la superficie transformada adoptan la valencia 1 o $2+$.

Estructuralmente, la posibilidad de existencia de pequeñas cantidades de $\mathrm{Al}^{2+}$ en la red polimérica de escorias aluminosas en fusión puede facilitar la retención de la coordinación tetraédrica, aún ante deficiencia de oxígeno, igual que en elevadas concentraciones de alúmina. Se sugiere también que la generación de $\mathrm{Al}^{2+}$ por una reacción, tal como se describe en la ecuación [7], proporciona una explicación alternativa para la efervescencia de oxígeno observada en las escorias líquidas. Esta hipótesis también facilita nuevas interpretaciones con respecto a la termodinámica y las propiedades físico-químicas de las escorias aluminosas.

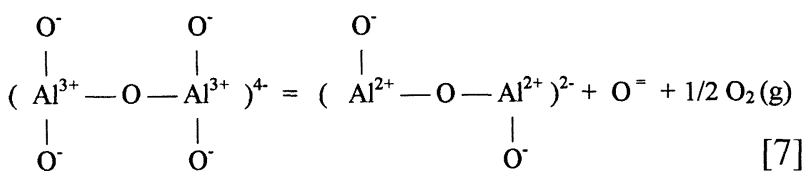

Una potencial explicación de los resultados de tensión superficial puede originarse en la combinación del hecho de que la reacción [7] propone la generación de dos especies aniónicas de un simple anión, con la posibilidad de que, gracias a la elevada actividad de la alúmina, se puedan facilitar reducciones en el estado de valencia de los iones de aluminio.

Se sugiere que la concentración superficial de especies aniónicas despolimerizadas, producidas por ejemplo por la reacción [7], contribuye al aumento de la concentración de uniones no saturadas en la región superficial y, consecuentemente, en la tensión superficial.

Cabe resaltar que la hipótesis del estado de valencia reducido para el aluminio es atractiva, dando una explicación común a las tendencias observadas en las propiedades físico-químicas del interior de la fase líquida, como por ejemplo la viscosidad, y de la región superficial.

\section{CONCLUSIONES}

Se determinaron la tensión superficial, densidad y viscosidad de escorias aluminosas. Los resultados están de acuerdo con la literatura y confirman una relación anómala entre la tensión superficial y la composición del sistema $\mathrm{CaO}-\mathrm{Al}_{2} \mathrm{O}_{3}$.

Se utilizó un modelo, propuesto previamente para interpretar aparentes discrepancias relativas a las capacidades de azufre en aluminatos de calcio, para explicar el aumento observado en la tensión superficial cuando se aumentó la concentración de alúmina. Se deduce que la presencia de pequeñas cantidades de $\mathrm{Al}^{2+}$ debe facilitar el mantenimiento del balance de cargas en coordinación tetraédrica en sistemas ricos en alúmina. Esta hipótesis es sugerente porque explica el aumento de la viscosidad y de la tensión superficial con los incrementos en las concentraciones de alúmina. Por lo tanto, respecto a esto se necesitan más investigaciones.

Se eluden también las interpretaciones en cuanto a la dependencia de las escorias aluminosas respecto de la presión parcial de oxígeno. Se sugiere que la observación de defectos estructurales relacionados con el oxígeno, como superradicales $\mathrm{O}_{2}^{-}$y/o efervescencia asociada, no debe restringirse a la fase $12 \mathrm{CaO}_{7} \mathrm{Al}_{2} \mathrm{O}_{3}$, como está ampliamente divulgado.

La caracterización de aluminatos binarios vítreos y la determinación de propiedades físico-químicas de aluminatos y escorias aluminosas son relevantes para el cuestionamiento de su modificación estructural y para el esclarecimiento de la influencia de los defectos estructurales relacionados con el oxígeno sobre la estructura superficial de estos materiales.

La combinación de estas investigaciones contribuye con evidencias adicionales a la elucidación de discrepancias respecto a sus propiedades físico-químicas y termodinámicas. Esto puede corroborarse con la optimización estructural de materiales cerámicos de aluminatos y de sus propiedades ópticoelectrónicas, así como de Termofosfatos, de elevada eficiencia de fertilización, producidos a partir de escorias metalúrgicas (21).Sus inferencias son también relevantes para el desarrollo de modelos coherentes para estimar propiedades físico-químicas de escorias metalúrgicas, tales como la basicidad óptica, conductividad eléctrica, densidad, viscosidad y tensión superficial. Tales estimaciones son importantes para el control de los fenómenos interfaciales que suceden en la metalurgia extractiva. A este respecto, se torna interesante cuestionar los efectos de la existencia de iones de aluminio en estado de valencia reducido en escorias aluminosas, y posiblemente alúmino-siliciosas, para el control de la escoria espumosa y para la separación de fases metal/escoria.

\section{REFERENCIAS}

(1) Askari, M. y Cameron, A. M. Can. Metall. Q., 30 (4), 1991: 207-212.

(2) Schrödinger, E. Ann. Phys., 46, 1915:413-416.

(3) Oliveira, F.A. Tesis de Doctorado, UMIST, Manchester, Agosto 1995: (a) Cap. 5 , (b) Cap. 3.

(4) Broadbent, C. et al. 4th Int. Conf. Molten Slags and Fluxes, ISIJ, Japan, 1992: 439-444.

(5) Kozakevitch, P. Phys. Chem. Proc. Metal. Int. Symp., Pittsburgh, Abril 1959: 97-116. 
(6) ZielinSKI, M. y SiKora, B. Hutnik, 41 (9), 1974: 433-437.

(7) Zielinski, M. y Sikora, B. Prace IMZ, 29 (3-4), 1977: 157-165.

(8) Mills, K.C. y KeEne, B. J. Int. Mat. Rev., 32 (1-2), 1987

(9) PoPEL, S.I. et al. Inst. Chern. Met. 12, 1971: 92-97.

(10) KeEne, B.J. Can. Metall. Q., 21(4),1982: 393-403.

(11) Hosono, H. y Abe, Y.; J. Am. Ceram. Soc., 70 (3) 1987: C38-C39.

(12) LuPIS, C.H.P. Chemical Thermodynamics of Materials, North-Holland, 1983 XIII: 392-395.

(13) Guggenheim, E.A. Surfaces of Simple Liquid Mixture. Clarendom Press, Oxford, 1952: 166-182.

(14) McMillan, P. y Piriou, B. J. Non-Cryst. Solids, (55), 1983: 221-242.
(15) Nurse, R.W. Brit. Ceram. Soc., (64), 1959: 323-332 y 409-411.

(16) Fine, H. A. Metall. Trans., Vol. 24B (10), 1983: 685-692.

(17) Cameron, A. M. Tesis de Doctorado, Imperial College, London, 1983: 133-135.

(18) Richardson, F.D. y KoR, G.J. JISI, Julio 1968: 700-704.

(19) CAMERon, J et al. JISI, 204 Dec., 1966: 1223-1228.

(20) French, T.M. y Somorjai, G.A. J. Phys. Chem., 74 (12), 1970: 2489-2495.

(21) Veiga, M.M. y Paschoal, J.O. Panorama do Setor de Materiais e suas relações com a Mineração, CETEM, Rio de Janeiro, 1991. 